\title{
El problema de las categorías y sus usos en la gestión de la diversidad en las escuelas
}

\author{
The Problem of Categories and their Uses \\ in the Construction of Diversity in Schools
}

\author{
Eva Martín Coppola \\ Universidad Complutense de Madrid
}

\section{RESUMEN}

Este artículo reflexiona sobre los problemas de representación de categorías nacionales a partir de la aplicación de un sociograma en escuelas con un elevado porcentaje de alumnos extranjeros. Analiza los presupuestos teóricos que llevan a utilizar el sociograma como herramienta de medición y construcción de la integración social en contextos multiculturales. El desajuste producido entre los resultados del test y la observación participante en las aulas genera una reflexión en torno a los problemas de representación y categorización implícitos en la interculturalidad como proyecto socioeducativo. Para finalizar se apuntan los límites en la aplicación de esta herramienta en contextos multinacionales segregados, y los problemas fenomenológicos que presenta la categoría "nacionalidad" en este contexto.

Palabras clave: Escuela, Sociograma, Integración intercultural, Redes sociales, Nacionalidad.

\section{SUMMARY}

This article analyzes the problems related to the representation of national categories through the implementation of a sociogram in schools with a high percentage of foreign students. We discuss the theoretical assumptions about the use of sociograms to measure and build social integration in multicultural contexts. The mismatch produced between the results obtained through this tool and the results provided by my participant observation in classes, contributes to problematise the issues of representation and categorization contained in the interculturality as an educational project. Finally, the article points the limits of the implementation of sociograms in multinational and segregated contexts as well as the problems presented by the category "nationality" in that context.

Key words: School, Sociogram, Intercultural Integration, Social Network, Nationality.

\section{INTRODUCCIÓN}

La concentración escolar de los hijos de familias inmigrantes en determinadas escuelas no ha hecho más que multiplicar el interés de las instituciones por conocer la dinámica de las relaciones en el ámbito escolar y, más concretamente, los procesos de integración relacional del que son objeto los estudiantes extranjeros (Banks 2006; 
Fernández-Enguita, Gaete y Terrén 2008; Alegre Casanovas 2008; Martínez Usarralde 2010). Informes y estudios académicos se han hecho eco de la concentración del alumnado extranjero en centros de titularidad pública y, dentro de estos, en determinados centros hasta el punto de producir verdaderos "guetos educativos" (Ponce Solé 2007; García Castaño y Rubio Gómez 2013). A partir de la libertad de elección del centro escolar, el cruce entre preferencias familiares y ofertas educativas ha tenido como consecuencia que a penas un $20 \%$ de escuelas cuente con más del $60 \%$ de alumnos extranjeros en la ciudad de Madrid, produciéndose concentraciones superiores al 50\% en determinados centros (CIDE 2012).

Se presupone que una escuela con un alto porcentaje de alumnos extranjeros va a presentar necesariamente dinámicas de inclusión o exclusión basadas en la nacionalidad de los estudiantes. Una de las mayores preocupaciones de la escuela es precisamente evitar la formación de grupos entre los escolares basados principalmente en la nacionalidad de los mismos y fomentar una interacción entre estudiantes de distintas nacionalidades. Este miedo a la existencia de "comunidades cerradas" en las aulas en las que el sujeto recurre a su nacionalidad como elemento de identificación y agrupación sería reflejo, según apuntaba el director de un centro educativo de Madrid, del "fracaso de la escuela pública en la integración de los inmigrantes". De esta preocupación deriva un creciente interés por los "mapas sociales" y el análisis de las redes intraescolares, basado en la creencia de que permiten capturar el nivel de convivencia en las aulas y por tanto posibilitan una planificación e intervención en las mismas a través de distintas actividades y programas.

La aplicación de los test sociométricos en contextos escolares como herramienta utilizada para estudiar la integración relacional de estudiantes tiene ya una larga trayectoria, facilitando la comprensión de parte de los problemas de integración social de determinados colectivos (Dodge et al. 1990; Kosker, Nakken, Pilj y Van Houten 2009; Martín 2011). Jacob Levy Moreno, fundador de la sociometría, definía el test sociométrico como una herramienta utilizada para saber quién configura el entorno inmediato de los sujetos y qué grupos se forman a partir de sus preferencias, permitiendo la observación y medición de la cercanía o distanciamiento entre los sujetos. Si bien el uso del sociograma se ha traducido en una multiplicidad de investigaciones donde se ha intentado medir la formación de grupos en función de distintas variables — sexo, edad, formación, etc.-, la diversidad nacional y cultural ha sido uno de los mayores polos de atracción para la aplicación de este método. El sociograma condensa por lo tanto la manera en que se trabaja y gestiona la diversidad desde la lógica de las instituciones.

El contraste de los resultados obtenidos a través de un sociograma con otras técnicas de investigación como es la observación de las relaciones en el aula muestra, sin embargo, las limitaciones en su aplicación. ¿Cuándo y por qué los resultados sociométricos no reflejan la realidad de las redes sociales existentes? ¿Por qué trabajar con sus presupuestos puede llevar a un error de planificación en la intervención educativa?

A través de una investigación basada en la observación participante en las aulas de institutos madrileños, se mostrará cómo los sociogramas, tanto en sus presupuestos teóricos como en los resultados que producen, no reflejan una determinada realidad sino que ayudan a construirla. El sociograma como modo de aproximación a las relaciones sociales no desvela la pluralidad y complejidad de dichas relaciones alcan- 
zando resultados diferentes a los que la realidad cotidiana apunta (Ibáñez 1986; Martínez Gutiérrez 1999).

\section{METODOLOGÍA}

A través de un convenio firmado entre la Consejería de Educación de la Comunidad de Madrid y una conocida $\mathrm{ONG}^{1}$ para la implantación de un programa de educación intercultural, se aplicó un test sociométrico en 80 centros públicos de educación secundaria, a clases de $1^{\circ}$ a $4^{\circ}$ de la ESO. A través de este convenio formé parte del equipo ejecutor de dicho programa y participé durante un año escolar en cinco de estas escuelas, con porcentajes de hijos de familias inmigrantes de entre el 70 y $90 \%$, de las que derivan los resultados empíricos disponibles para esta investigación ${ }^{2}$. Una de las principales características de estos centros es la de tratarse de escuelas con un elevado porcentaje de alumnado extranjero, hasta el punto de ser consideradas como escuelas segregadas por el efecto huida de los alumnos españoles.

El objetivo de la ONG era intervenir en estas escuelas con el fin de desarrollar la interculturalidad, tal y como viene recogido en el Plan Estratégico de Ciudadanía e Integración 2011-2014: una situación real o deseada, en la que personas y grupos con nacionalidades y referentes culturales distintos conviven, es decir, dialogan, se relacionan en igualdad de condiciones, cooperan y colaboran, se mezclan y se respetan, en un mismo entorno físico. La escuela es señalada en este plan como lugar de promoción y desarrollo de la interculturalidad, con el fin de prevenir las situaciones de conflicto social, los procesos de discriminación, y en último extremo, el racismo y la xenofobia (Banks 2006; Ballesteros Velázquez y Gil Jaurena 2012). El querer aplicar dicho programa prioritariamente en escuelas con elevado porcentaje de alumnos extranjeros demuestra cómo la categoría de interculturalidad queda reducida a "asunto de o para migrantes" en lugar de abarcar la sociedad en su conjunto (Giménez 2010).

Me informé de la forma en que podía participar en dicho proyecto con el fin de valorar cómo entienden e implementan la interculturalidad desde las instituciones públicas. La organización, con problemas de personal para atender a la totalidad de las escuelas, veía en mi participación voluntaria la posibilidad de ampliar sus intervenciones; los centros escolares hallaban a alguien que se encargase de los talleres; y por mi parte yo obtenía la autorización para acceder a las escuelas y analizar el programa implementado y sus consecuencias. El principio de reciprocidad e intercambio entre los distintos actores e intereses quedaba, de esta forma, resuelto (Del Olmo 2010). Fue así como, en el transcurso de un año escolar, participé, de octubre a junio, en un programa sobre interculturalidad en los institutos a través de la cooperación establecida con una ONG de larga trayectoria en la intervención en el mundo educativo.

\footnotetext{
${ }^{1}$ El nombre de esta organización es mantenido en el anonimato por expresa voluntad de sus responsables.

${ }^{2}$ Los cinco centros se encuentran en el distrito de Tetuán, Hortaleza, Vallecas, y Centro de la ciudad de Madrid. Se trata de centros de educación secundaria públicos que se acogieron a este programa por su elevado porcentaje de alumnado extranjero. Este programa tienen un doble objetivo: evaluar la situación presente a través de un sociograma, e intervenir con el fin de construir un modelo de convivencia intercultural.
} 
Mi entrada en los institutos respondía a una doble estrategia: por un lado me presentaba como miembro de una organización que venía a enseñar y aplicar los talleres sobre interculturalidad, y por otro lado quería analizar, como investigadora, el programa que yo misma aplicaba. Mi doble rol, el de miembro de la ONG y el de investigadora en una institución escolar, me permitió considerar los talleres como micropolíticas de intervención y observar los resultados que producían en los sujetos intervenidos, abriéndome la posibilidad de contrastar dos fuentes diferenciadas de recogida de información:

a) Las actividades y material empleado por la ONG junto a las fuentes escritas de los estudiantes como resultado de las actividades recogidas al final de cada clase.

b) Las fuentes orales a través de la recogida de relatos, conversaciones y charlas cotidianas producidas en clase $-\mathrm{y}$ anotadas en el cuaderno de campo-, ayudándome a interpretar los resultados de los escritos a partir de lo registrado en el cuaderno de campo.

La necesidad constante de alternancia de roles en la investigación y de contraste entre distintas fuentes diferenciadas de recogida de información, otorgan al trabajo de campo un carácter complejo. Pero es precisamente dicha complejidad, consustancial a la etnografía escolar, la que permitió alcanzar los resultados de esta investigación (Díaz de Rada 2013).

Frente a los resultados producidos por el test sociométrico, la observación, las anécdotas surgidas en el desarrollo de las clases y la recogida de relatos, ayudan a interpretar y comprender los resultados "reales" de las actividades, o al menos, a contrastar dos fuentes diferenciadas de recogida de información: lo que escriben con lo que dicen, el discurso estructurado con el discurso espontáneo, dos formas de transmitir y construir conocimiento.

Pero antes de adentrarnos en los resultados empíricos resulta necesario realizar una aproximación crítica de los presupuestos teóricos en que se sustenta el sociograma como herramienta de aproximación e intervención en las escuelas.

\section{UNA LECTURA CRÍTICA DE LA APLICACIÓN DE SOCIOGRAMAS EN LAS ESCUELAS}

El sociograma es considerado, por muchos, como un instrumento pertinente para la evaluación de la convivencia, encaminado a la prevención e intervención escolar (Gázquez Linares et al. 2007). Para intervenir en la escuela es necesario empezar por conocer las problemáticas existentes entre los distintos grupos sociales, la formación de las redes de amistad, las fracturas y los grupos excluidos con el objetivo de ajustar la intervención a la resolución de problemas. Dadas las características de los centros escolares con elevados porcentajes de alumnado extranjero, la organización presupone la nacionalidad de los alumnos como elemento de fractura y/o cohesión grupal. ¿Existen prejuicios o enemistades manifiestas entre los distintos grupos nacionales? Tal era la pregunta que la ONG y la escuela buscaban resolver: conocer la realidad existente en las aulas y, a partir de ahí, producir un nuevo mapa relacional. Pero, ¿es el sociograma la herramienta apropiada para captar las conexiones socio-afectivas de los estudiantes? ¿Es la nacionalidad una variable adecuada a considerar como elemento de estructuración de grupos? La aplicación del sociograma viene precedida de unos presupuestos teóricos a partir de los cuales se desvela la lógica de actuación de las instituciones en la gestión de la diversidad, sobre la base de la nacionalidad. 


\section{LA NECESIDAD PROGRAMÁTICA}

La vieja imagen del aula como microsociedad (Parson 1976) convierte los centros escolares en antesalas donde surge y también se programa el tipo deseable de relaciones e interacciones sociales: "La escuela es uno de los pocos ámbitos de socialización en que es posible 'programar' experiencias de contactos entre sujetos diferentes, de encuentros que permitan enriquecerse con la cultura de las otras personas" (Tedesco 1995). Bajo esta premisa, las posibles dificultades de integración de los extranjeros, las actitudes o comportamientos discriminatorios, el racismo o el aumento de prejuicios y estereotipos hacia los grupos minoritarios son elementos que pueden detectarse tempranamente e intervenirse para su resolución. La escuela debe ser capaz de generar vínculos que permitan incorporar a los individuos y grupos asegurando una cohesión social. El concepto de amistad intercultural es fomentado como un ideal de integración relacional en contextos multi-culturales y supone la intervención de una institución en el fomento y dirección de las afinidades y lazos afectivos "deseables".

¿Puede favorecer la escuela la permeabilidad intergrupal? Si bien la escuela se presenta como un centro de interacción y participación ciudadana, que en principio debería facilitar la integración de los distintos colectivos, no resulta un espacio neutro a la hora de promover las relaciones igualitarias (Dubet 2008; Fernández Enguita 2008; Poveda et al. 2012). El hecho mismo de que la escuela arrastre importantes problemas de segregación escolar es reflejo de las desigualdades que se establecen en su seno, imposibilitando, ya desde la base, un contacto equilibrado entre los distintos grupos sociales. En efecto, la escuela tiene una doble vertiente: a través de ella se organiza y crea un tipo de sociedad; sin embargo, crea, al mismo tiempo que refleja, las dinámicas sociales y desigualdades existentes en el sistema. El propio clima escolar puede, además, estimular la competición premiando a quienes mejores resultados académicos obtengan, por lo que la igualdad de estatus sería igualmente cuestionada; la cultura académica sigue representando un espacio de confrontación y escenificación de las relaciones de poder (Solbes et al. 2011).

\section{LAS CATEGORÍAS NACIONALES Y CULTURALES}

El análisis de este sociograma está ligado al interés de la ONG e instituciones, y es un reflejo de cómo piensa y trabajan la interculturalidad poniendo en evidencia el modo de mirar las interacciones en el aula y construyendo una particular visión de lo que en ella acontece. La utilización de la nacionalidad como variable interpretativa tiene una implicación metodológica importante ya que excluye gran parte de los procedimientos para el análisis de los resultados que se basan en la producción de categorías de individuos distinguidos en función del estatus de elección y el estatus de rechazo (Arruga 1983). ¿Obtendríamos los mismos resultados si partiésemos de otro tipo de categoría? Toda representación de un mapa social, sin ser cierta o falsa, representa tan solo un modo particular y concreto de mirar dicha realidad. Es la mirada la que hace de la percepción un modo de ver específico y que da una forma particular a los datos percibidos construyendo distintas realidades. ¿Por qué partir de la nacionalidad para llegar a la interculturalidad? A través del sociograma, se produce un proceso de "categorización" de las relaciones escolares: (1) asignando categorías nacionales a los sujetos, (2) presupo- 
niendo que a dichas categorías nacionales le corresponde una determinada cultura, y (3) promoviendo la interacción entre las distintas categorías - nacionales y culturalescomo único modelo deseable de integración social escolar.

Realizar un sociograma desde una perspectiva grupal y nacional supone por lo tanto construir una determinada realidad y provocar efectos sobre ella. Analizar las relaciones escolares a través de dichos enunciados no supone afirmar si son ciertos o falsos, sólo podemos decir de ellos si son afortunados o no afortunados en virtud de que aseguren su eficacia en la producción de efectos en la realización de aquello que se pretende con ellos (Garay, Íñiguez y Martínez 2003).

\section{LAS REDES SOCIALES Y SU CONTEXTO}

Los estudios que trabajan el concepto de "amistad intercultural" en las aulas realizan su análisis con base a dos categorías de estudiantes claramente diferenciadas, generalmente representadas por el grupo mayoritario, como un todo homogéneo, frente a los grupos minoritarios — cambiantes según el contexto y la época- (Arnáiz y De Haro 2003). Estos estudios tratan de describir el grado de aceptación del grupo mayoritario de los grupos minoritarios, y el grado de integración relacional de los grupos minoritarios con el mayoritario. Las tendencias generales que se han detectado apuntan a la preferencia de las personas a relacionarse con su propio grupo nacional y visibiliza las dificultades de una interacción entre grupos mayoritarios y minoritarios bosquejando un mapa relacional fragmentado por razón de la procedencia (Fernández Prados y Soriano Ayala 1997; López Castro 2000).

Ahora bien, el tradicional uso de estas categorías por parte de los sociogramas no puede ser replicado en las escuelas objeto de estudio: las escuelas segregadas obligan a trascender las categorías tradicionales, tanto las relativas a la nominación de los grupos - grupo mayoritario/grupos minoritarios- como a la tipología resultante de su interacción. ¿Qué ocurre con las relaciones de amistad entre grupos minoritarios? En este sentido resultaría útil aplicar el concepto de integración relacional de los inmigrantes de Federico de La Rúa (2004), que permite caracterizar los tipos de micro estructuras relacionales presentes en espacios transnacionales donde se toman en consideración tanto los lazos locales — cuando se trate de vínculos con la sociedad de acogida-, como los lazos nacionales — aquellos establecidos con personas del mismo origen - o los transnacionales — cuando se trate de relaciones con personas de distintos orígenes- La ventaja de utilizar esta tipología es que considera la adaptación de los inmigrantes, no sólo en función de los vínculos que mantienen con la comunidad de origen o la sociedad de acogida, sino también de aquellos relativos a otras comunidades transnacionales. La interacción intercultural que se juega en este escenario es, por tanto, diferente y las categorías de aplicación deberían, de este modo, variar en lugar de mantener la estructura relacional bi-direccional — sociedad de origen, sociedad de acogida (Berry 1997).

El sociograma fue aplicado sin tomar en consideración estas precauciones metodológicas, produciendo unos mapas sociales que fueron utilizados para evaluar el estado de la convivencia e intervenir en los centros escolares. ¿Qué nivel de integración relacional se produce en estas escuelas? 


\section{LOS RESULTADOS SOCIOMÉTRICOS}

A continuación presentamos los resultados, tal y como fueron obtenidos por la ONG, de los mapas sociales más representativos de las 68 clases donde se realizaron. Son tres los escenarios encontrados, que pueden resumir los diferentes modelos de integración relacional que se producen en las aulas con elevados porcentajes de alumnado extranjero. Con un porcentaje de alumnado extranjero matriculado similar, las únicas variaciones existentes son las relativas a la composición de las aulas, es decir, el número de alumnos y las nacionalidades presentes en las mismas ${ }^{3}$.

\section{LA NACIONALIDAD COMO ELEMENTO DE AGRUPACIÓN}

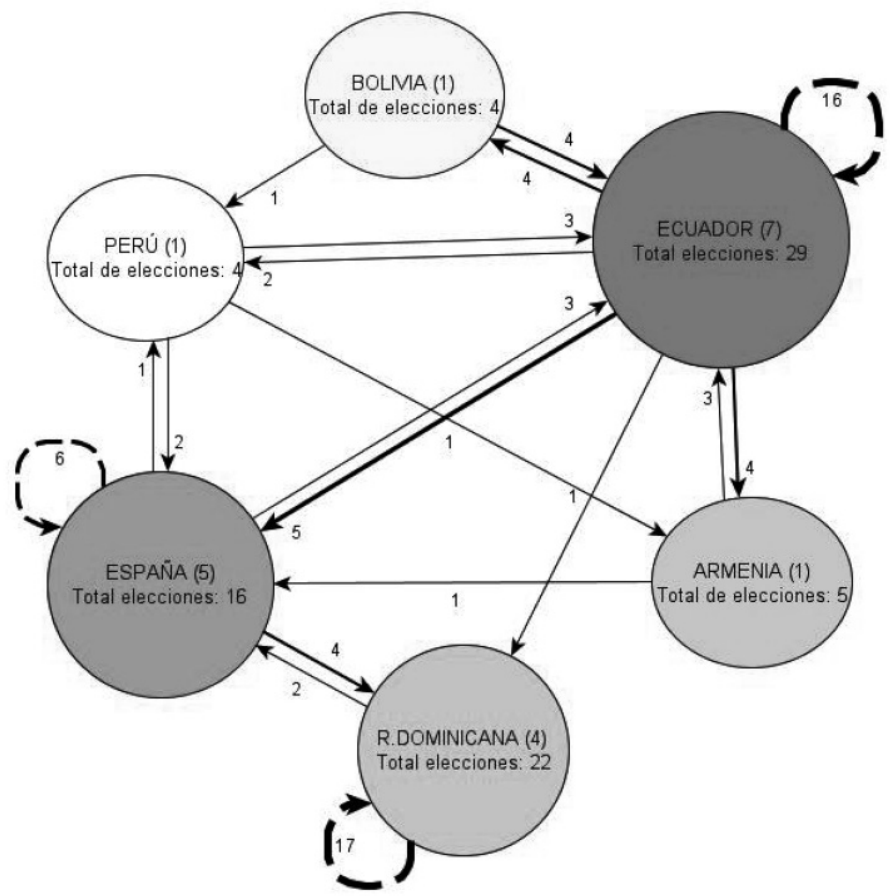

ILUSTRACIÓN 1.-Representación de los resultados del sociograma por nacionalidades.

Fuente: elaboración propia.

\footnotetext{
${ }^{3}$ Los tres gráficos se componen de los distintos grupos nacionales presentes en el aula, el número de personas que componen dicho grupo, el total de elecciones que recibe y la procedencia de las elecciones por grupos nacionales. Los círculos representan los distintos grupos nacionales. Dentro de cada ć́rculo, entre paréntesis, el número de personas de dicha nacionalidad y el total de elecciones que recibe el grupo. La dirección de las flechas indica el número de elecciones que realiza y recibe cada grupo nacional de los demás. Las fechas circulares representan el número de autoelecciones de amistad que realiza cada grupo hacia sus propios miembros.
} 
En el primer escenario, la nacionalidad aparece como un elemento de agrupación e identificación importante a la hora de formar grupos de amistad sin suponer por ello "comunidades cerradas", puesto que existen entre sus miembros interacciones con otras personas de nacionalidades diferentes.

\section{PREFERENCIA HaCia UN MisMo GRUPO NACIONAL}

El siguiente escenario muestra una situación bien diferente, la existencia de una preferencia generalizada de la mayoría de grupos por una misma nacionalidad, en este caso la española, que se convierte en el grupo de referencia. La centralidad sociométrica del grupo no supone, sin embargo, la desconexión del resto; todos los grupos están conectados entre sí y presentan amigos del resto de nacionalidades.

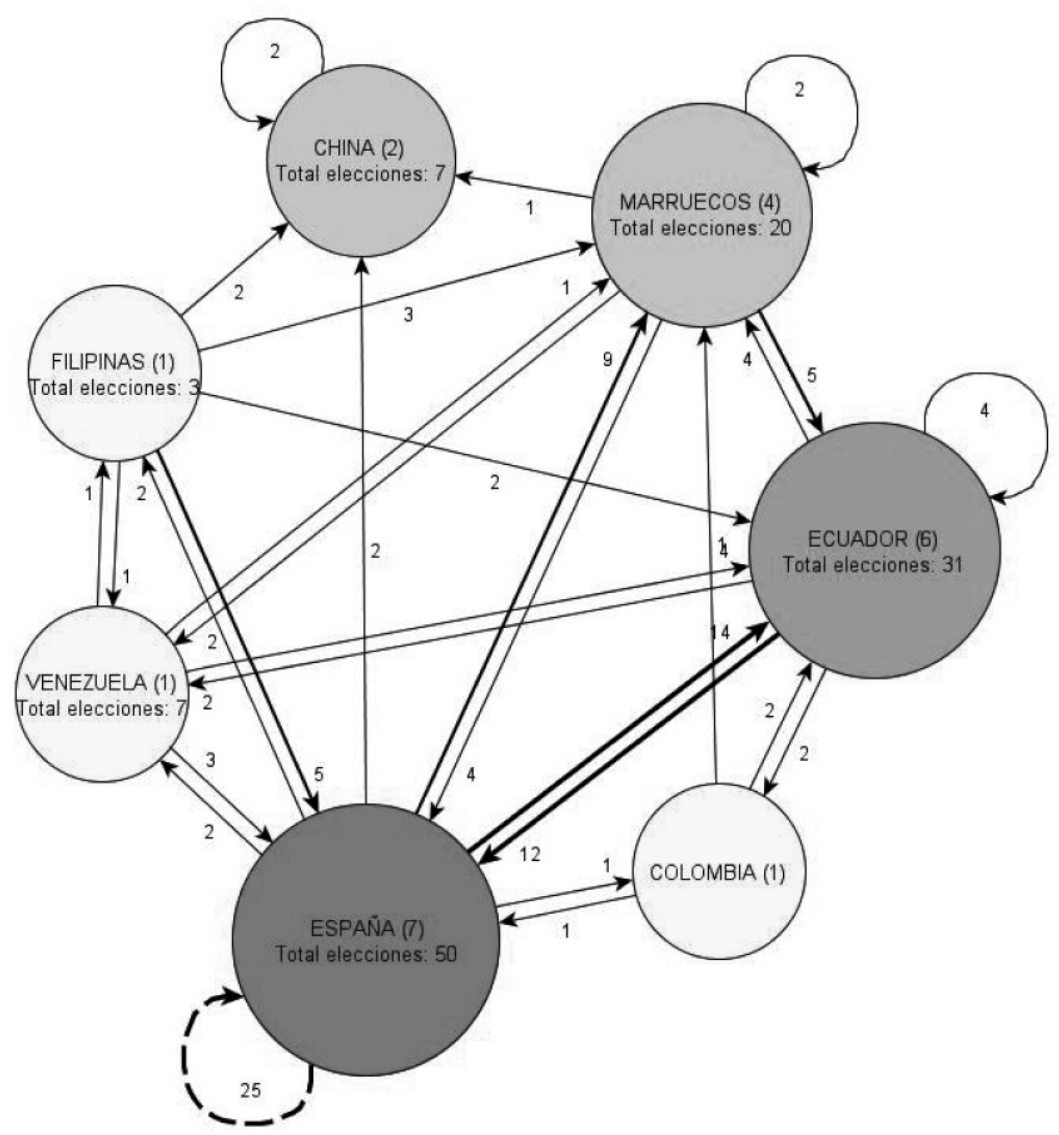

ILUSTRACIÓN 2.--Representación de los resultados del sociograma por nacionalidades. Fuente: elaboración propia. 


\section{RELACIONES MULTINACIONALES}

En este último escenario, la nacionalidad desaparece como elemento de identificación a la hora de observar los vínculos de amistad ya que todos parecen tener más amigos de otras nacionalidades que de la suya propia, sin tampoco demostrar preferencia por ninguna en particular. De modo que se dan grupos completamente diversos desde el punto de vista de la nacionalidad.

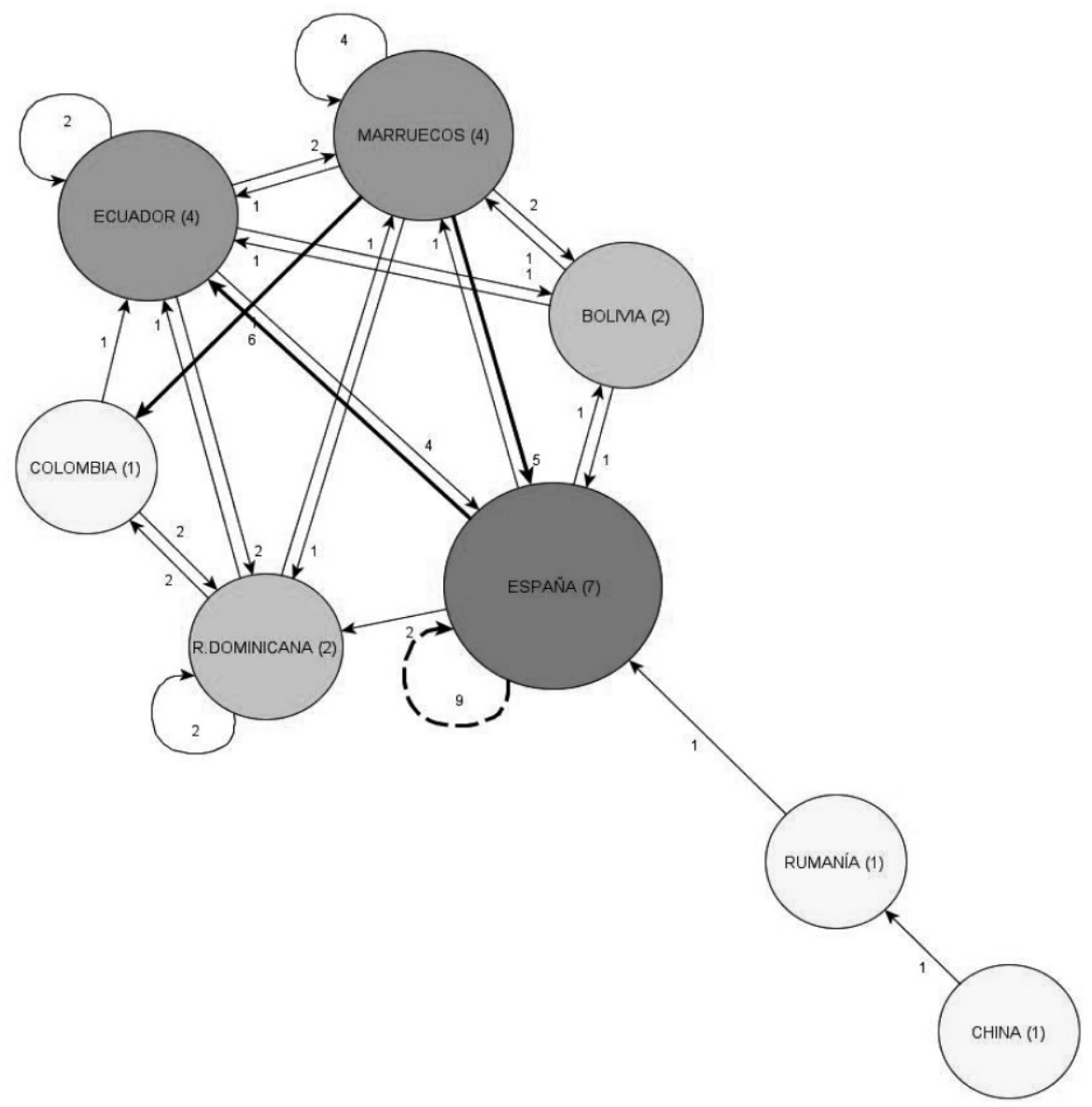

ILUSTRACIÓN 3.-Representación de los resultados del sociograma por nacionalidades. Fuente: elaboración propia.

Estos tres escenarios mostrados no permiten extraer ninguna tendencia clara. Los resultados de los cuestionarios sociométricos tan solo apuntan una conclusión: la composición de las aulas, el número relativo de grupos nacionales y su peso cuantitativo dentro del aula produce tantos modelos de relación como posibilidades existen. Se pueden encontrar agrupaciones en torno a la nacionalidad cuando se da la existen- 
cia de dos grandes grupos en una clase, es decir, por ejemplo, frente a una mayoría de ecuatorianos y dominicanos, los estudiantes encuentran una posibilidad de agrupación casi automática; sin embargo, frente a la existencia de aulas con una presencia plural de distintas nacionalidades no existe agrupación alguna de los sujetos con respecto a su nacionalidad en la formación de grupos. Se podría por tanto aventurar la hipótesis de que al aumentar el número de grupos nacionales en el aula y desaparecer las mayorías, la amistad intercultural aumenta. Las relaciones endogámicas no son connaturales a los sujetos sino que dependen de la composición de las aulas.

Sin embargo, estos resultados no recogían el aspecto más relevante de todos para la comprensión e interpretación de los resultados: las dificultades de identificación y adscripción de los sujetos a las categorías utilizadas por el sociograma. El sociograma clasifica a la población a partir de rasgos atribuidos "objetivamente" como es la nacionalidad, sin embargo no permite la consideración de la identificación de los sujetos con respecto a dicha categoría. Estos resultados fueron matizados a través de la observación participante.

\section{LOS VÍNCULOS OBSERVADOS DURANTE LA APLICACIÓN DEL SOCIOGRAMA}

En efecto, durante el desarrollo del sociograma en las distintas clases pude comprobar hasta qué punto la atribución de la nacionalidad de los estudiantes, ya fuera propia o ajena, se convertía en un verdadero debate relativo al sentimiento de pertenencia. El test sociométrico lo administré yo misma para poder escuchar in situ los comentarios realizados al respecto, aclarar dudas y observar las posibles reacciones.

En este apartado se recogen las reacciones de los alumnos ante la aplicación del sociograma y una reflexión en torno a los problemas fenomenológicos de la categoría nacionalidad en la aplicación del mismo. La fuente documental procede de las anotaciones realizadas en el cuaderno de campo. ¿Qué limitaciones presenta el sociograma, tal y como fue aplicado, como herramienta de análisis y descripción de la realidad?

Antes de comenzar a contestar las preguntas, los alumnos debían rellenar un pequeño recuadro con su nombre, apellidos, edad y nacionalidad. Si en la casilla "nacionalidad", los alumnos tienen dificultades en contestar, ¿Qué está ocurriendo? A raíz de la distribución del sociograma sucedió algo completamente inesperado en tanto en cuanto no había sido previsto el cuestionario.

\section{1. “¿QUÉ ES LA NACIONALIDAD?"}

Tras escribir sus nombres y edades, comenzaron a surgir algunas manos levantadas que demandaban aclaraciones en torno al significado de "nacionalidad". Este hecho paralizó el desarrollo normal de la cumplimentación del cuestionario, generando una interesante situación en la cual quedaron patentes, como punto de partida e interés de la investigación, los distintos significados e importancia otorgados a la nacionalidad entre los estudiantes.

La intervención de unos dio lugar al cuestionamiento de otros, y así sucesivamente, como si se contagiaran y empezaran a mostrar dudas sobre qué poner: 
- “¿Nacionalidad es dónde naces o de dónde vives? Yo llevo 6 años aquí, ¿Pongo que soy español o ecuatoriano?"

- «Tienes que poner lo que pone en tus papeles, o la que tú crees? Yo tengo la nacionalidad española pero me considero marroquí. ¿Qué pongo?»

- "iLa nacionalidad es como decir la cultura y eso?"

- "Yo tengo la nacionalidad boliviana pero me fui de ahí cuando era un bebé, ¿La pongo o puedo decir que soy más española?"

- "¿Se pueden poner varias a la vez o eso es imposible?" "Yo es en el tercer país que vivo, ¿Pongo todas?" [Alumnos de clase].

Fueron ellos mismos los que se respondían, generando un interesante debate en torno al significado que otorgaban al concepto de "nacionalidad", en el que yo me limité a escuchar, anotar sus comentarios y darles la palabra. Los alumnos aprovecharon para cuestionar el concepto y ofrecer o mostrar formas alternativas de adhesión. ¿Qué significado adquiere la nacionalidad, que lo ponga en "tus papeles", que los demás te asignen una o que la sientas como propia? Estas intervenciones dan muestra de la variedad de acepciones del término "nacionalidad" para los estudiantes.

Así, la nacionalidad, puede remitir en primer lugar, a una definición más jurídica, esto es, la pertenencia de una persona a un ordenamiento jurídico concreto, el vínculo del individuo con un Estado determinado que genera derechos y deberes recíprocos. Este concepto de nacionalidad se mezcla, sin embargo, con el de nacionalidad como situación social que une a un individuo con un grupo en virtud de una vida en común. A ello se suman dos concepciones más, la nacionalidad como adscripción cultural —aludiendo a las distintas costumbres, prácticas, lenguas, ritos y usos que determinados grupos pueden mantener en la sociedad de llegada- y la nacionalidad como sentimiento de pertenencia. Al no prevalecer un significado sobre otro, no se podía alcanzar un consenso relativo a una definición que permitiese otorgar el mismo valor a las diferentes respuestas del sociograma.

\section{LA ALTERNANCIA DE REGISTROS}

Frente a la pregunta, menciona a tus tres mejores amigos y dime cuál es su nacionalidad, muchos estudiantes no sólo cuestionaron su nacionalidad, con base en los distintos significados expuestos, sino también la de sus compañeros, que en ocasiones mostraron cierto desconcierto. Las actitudes en las aulas se caracterizaron por la existencia de una solidaridad derivada de compartir una misma situación, es decir por la capacidad de alternar varios registros a la vez, en la que todos son de alguna manera cómplices en el reconocimiento y libertad de elección de cada cual. La situación comunicativa se produce en un contexto de consenso y aceptación social donde los distintos sujetos encuentran los incentivos necesarios para desarrollar una actitud de atribución y asignación de categorías nacionales subjetiva.

A: "¿Eh, tú de dónde eras?"

B: "Soy ecuatoriano. Bueno y español."

A: "Qué prefieres que te ponga, elige."

B: "Da igual... no sé si puedes pon las dos Y si no pues... yo que sé." [Alumnos de clase]. 
No se trata exclusivamente de una cuestión subjetiva basada en el libre sentimiento de pertenencia, sino que existe un componente fundamental, el reconocimiento de dicha pertenencia, es decir que los otros reconozcan la libertad de elección y los diferentes significados otorgados a la nacionalidad. Esta proximidad sociológica favorece una condición fundamental para la comunicación no-violenta donde se produce, entre los interlocutores, la intercambiabilidad y la garantía contra la amenaza de ver las razones subjetivas de uno reducidas a causas objetivas (Bourdieu y Wacquant 1992). Los estudiantes muestran una actitud de solidaridad fundada en el sentimiento de compartir "una misma situación": la capacidad de alternar con varios registros a la vez, tal y como queda patente a continuación. Se trata de un ejercicio de verdadera habilidad en la medida en que constituye una fuente de acción del sujeto frente a las categorías:

- "Yo creo que tenemos que poner de dónde nos sentimos, ¿No?"

- "¿Por qué es tan importante poner la nacionalidad? Mis padres por ejemplo nacieron en un sitio, vivieron en otro y ahora están aquí, así que es como si tuvieran 3 nacionalidades."

- "Yo depende de dónde estoy y con quién, me siento de un sitio u otro. Ahora en particular me da igual.» [Alumnos de clase].

A esto hay que añadir parte del desconocimiento o la sorpresa que unos mostraban cuando escuchaban hablar a sus compañeros sobre su nacionalidad, bien porque habían supuesto una que no correspondía con la que estaban oyendo, bien porque, al no concederle importancia, no se habían molestado en preguntárselo. En este sentido, es el sociograma el que crea el problema e introduce la necesidad y obligación de identificación de los sujetos a partir de una nacionalidad.

En el acto de clasificación que requiere el sociograma, tiene lugar una triple negociación, la de uno consigo mismo tratando de buscar posibles identificaciones, la que mantiene con los compañeros que le atribuyen una u otra, esto es, el reconocimiento por los demás de la elección de cada uno, y por último, la negociación con el entorno, es decir, las referencias inmediatas de las demás personas y su capacidad para valorar positivamente este ejercicio de construcción de identificaciones ${ }^{4}$ :

- "Pero qué es eso de gitano, ¿Tú no eras español? ¿Dónde está ese país?" «Pero qué vas a ser español si tienes cara de moro!" "¿Y tú qué sabrás lo que es una cara de moro?" “iAh!, ¿Que eres moro? ¡Yo pensaba que eras ecuatoriano!» "Tú sí que no te enteras de nada..."

- "Profe, no le hagas caso a Fadua, ella es marroquí pero ahora le da por decir que ella es española, y que no conoce Marruecos, pero su hermano dice que es mentira."

- ¿¿Y qué más da? ¿Que cada uno sea de donde le dé la gana! ¿En qué te molesta?” [Alumnos de clase].

\footnotetext{
${ }^{4}$ La perspectiva interaccionista es aquí útil, ya que representa la relación dialéctica permanente entre cómo nos vemos a nosotros mismos y queremos ser vistos por otros, y cómo nos ven/categorizan los demás (imagen pública) en el proceso de construcción de la identidad social (Jenkins 2000).
} 


\section{LOS VÍNCULOS EMOCIONALES}

En la mayoría de los casos, la nacionalidad es vivida entre los estudiantes como un posicionamiento de carácter emotivo, tal y como quedó recogido en el diario de campo:

- "Mi abuela siempre me dice que uno es de donde es su familia que le quiere, que para qué vas a ser de donde no te quieren"

- "Pues yo ya me considero de aquí, ¿No ves que tengo amigos de todas la nacionalidades?"

- "Si todos los peruanos son como mi amigo, yo quiero ser de nacionalidad peruana"

- "Yo prefería la de antes (nacionalidad) que la de ahora, tenía más amigos".

- "Yo antes era de [...] pero desde que se vino toda mi familia a España yo ya me siento más española" [Alumnos de clase].

La aparición de identificaciones afectivas/emotivas refleja la manera de relacionarse del individuo con las categorías sociológicas más genéricas. Es precisamente en esos niveles relacionales donde finalmente se sitúa el sentimiento de pertenencia a un lugar o a un grupo social. No se trata pues de una adscripción objetiva ya constituida, sino del agrupamiento como un proyecto que se construye a través de identificaciones afectivas. Mientras que algunos — profesores y miembros de la ONG - se empeñan en hablar de la nacionalidad como si ésta tuviera vida propia y la fuerza suficiente para determinar las lealtades y comportamientos, observamos cómo en muchos casos para estos estudiantes es más importante una identificación afectiva. En efecto, la "nacionalidad" aquí hace referencia a la capacidad de asociarse emocionalmente con otra persona, categoría o colectividad.

\section{LA AMBIVALENCIA DE UN CONCEPTO: NACIONALIDAD JURÍDICA, SUBJETIVA Y SOCIAL}

El desconocimiento inicial relativo a la nacionalidad de muchos compañeros, dudando o confundiendo en algunos casos unas con otras, y la dificultad de adscripción a una nacionalidad por parte del mismo sujeto, hizo presagiar un importante margen para la ambivalencia en la atribución de nacionalidades.

En efecto, tanto en la primera pregunta — donde se preguntaba por la nacionalidad del sujeto- como en la atribución de la nacionalidad a los amigos elegidos, se demostró que la lista de alumnos que los profesores tenían para cada clase no reflejaba la percepción de los propios estudiantes (ver tabla 1). En ocasiones se sobrerrepresentaba un colectivo, otorgando una mayoría nacional a quien no la tenía, o eliminando un colectivo adjudicándole una nacionalidad distinta. Esto, junto a una multitud de tachones en la casilla de "nacionalidad", donde en ocasiones se llegaba a cambiar por dos y tres veces la misma, aconsejaba mantener cierta prudencia con los resultados obtenidos a partir del sociograma. 
TABLA 1

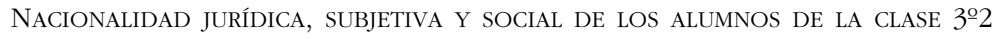

\begin{tabular}{|c|c|c|c|c|}
\hline № & $\begin{array}{l}\text { Apellidos } \\
\text { y Nombre }\end{array}$ & $\begin{array}{l}\text { Nacionalidad } \\
\text { Jurídica }\end{array}$ & $\begin{array}{c}\text { Nacionalidad } \\
\text { Subjetiva }\end{array}$ & $\begin{array}{c}\text { Nacionalidad } \\
\text { Social (atribuida) }\end{array}$ \\
\hline 1 & $* * * *$ & Española & Ecuatoriana & Ecuatoriana \\
\hline 2 & $* * * * *$ & Española & Española & Española \\
\hline 3 & $* * * * *$ & Ecuatoriana & Ecuatoriana & Ecuatoriana \\
\hline 4 & $* * * * *$ & Ecuatoriana & Ecuatoriana & Española \\
\hline 5 & $* * * *$ & Dominicana & Española & Dominicana \\
\hline 6 & $* * * * *$ & Ecuatoriana & Ecuatoriana & Ecuatoriana \\
\hline 7 & $* * * *$ & Ecuatoriana & Española & Ecuatoriana \\
\hline 8 & $* * * *$ & Marroquí & Marroquí & Marroquí \\
\hline 9 & $* * * * *$ & Española & Marroquí & Marroquí \\
\hline 10 & $* * * * *$ & Marroquí & Española & Marroquí \\
\hline 11 & $* * * *$ & Marroquí & Marroquí & Española \\
\hline 12 & $* * * * *$ & Búlgara & Búlgara & Búlgara \\
\hline 13 & $* * * * *$ & Ecuatoriana & Ecuatoriana & Ecuatoriana \\
\hline 14 & $* * * *$ & Española & Rumana & Española \\
\hline 15 & $* * * * *$ & Española & Española & Española \\
\hline 16 & $* * * *$ & Española & Española & Española \\
\hline 17 & $* * * * *$ & Ecuatoriana & Ecuatoriana & Dominicana \\
\hline 18 & $* * * *$ & Ecuatoriana & Española & Ecuatoriana \\
\hline 19 & $* * * * *$ & Polaca & Polaca & Polaca \\
\hline 20 & $* * * *$ & Ecuatoriana & Ecuatoriana & Española \\
\hline 21 & $* * * * *$ & Dominicana & Dominicana & Dominicana \\
\hline 22 & $* * * * *$ & China & China & China \\
\hline 23 & $* * * * *$ & Marroquí & Española & Ecuatoriana \\
\hline \multirow[t]{2}{*}{24} & $* * * * *$ & China & China & China \\
\hline & & & $\begin{array}{l}\text { (33\% de } \\
\text { ambivalencia) }\end{array}$ & $\begin{array}{l}\text { ( } 29 \% \text { de } \\
\text { ambivalencia) }\end{array}$ \\
\hline
\end{tabular}

FUENTE: Elaboración propia. 
En las intervenciones producidas en el aula queda reflejada la ambivalencia ${ }^{5}$ con la que estos jóvenes se relacionan con la idea de nacionalidad. Utilizando el término acuñado por Zigmunt Bauman, podríamos decir que estamos frente a la constitución de identificaciones ambivalentes. Para Bauman, el individuo confiere al mundo una estructura y un orden a partir del lenguaje y las clasificaciones. A través de la interiorización de este marco común, el mundo aparece ante nosotros como coherente, limitando el surgimiento de lo imprevisible o inclasificable. La ambivalencia, sin embargo, muestra los límites de este aparente orden, dando lugar a la aparición de la indeterminación y la contingencia:

La ambivalencia es la consecuencia inevitable de todos los proyectos de clasificación clara y exhaustiva [...] resulta de la asunción de que la gente o muchos rasgos pueden ser separados en un dentro/fuera, beneficioso/dañino, relevante/irrelevante. Cada clasificación engendra ambivalencia: no la habría si no fuera por la visión que implica necesariamente toda búsqueda de orden (Bauman 1990: 184).

A través de esta ambivalencia, las personas se alejan del determinismo, lo que les permite reflexionar sobre las categorías y visiones dicotómicas. Son varios los autores que han visto en la ambivalencia la posibilidad, la excusa o el punto de partida para observar la sociedad, sus grupos o instituciones desde otra mirada, en la que tengan cabida los comportamientos, acontecimientos y experiencias sociales excluidas por los sistemas de clasificación (Lamo de Espinosa 1989; Cousin 2002; Rovira Kaltwasser 2012).

Existen dos enfoques, haciendo uso de la vieja polaridad epistemológica de lo emic frente a lo etic, que nos permiten comprender la naturaleza de esta quiebra entre las categorías e identificaciones. El primero —etic- es una descripción de hechos o atributos observables; se toman las variables sociodemográficas tradicionales, como por ejemplo la nacionalidad, la edad o el sexo como elementos de identificación de los cuales se derivan determinados vínculos sociales. Las primeras preguntas que formularemos, serían seguramente del tipo: ¿De dónde eres? ¿Dónde vives? ¿Qué edad tienes? ¿En qué trabajas? De esta manera estamos, en buena parte, realizando un proceso de categorización social básico que nos posibilita la identificación de una persona con su entorno social ¿Pero qué ocurre cuando la categoría sexo da lugar a la construcción del género, cuando la categoría nacionalidad nos impide ver posibles mestizajes, o que el lugar de nacimiento no muestre el itinerario de movilidad de un individuo?

El segundo enfoque - emic- es una descripción en términos significativos para el agente que la realiza, se circunscribe a las visiones y sentimientos de pertenencia narradas por los propios sujetos. Éstos pretenden explorar cómo se producen y construyen los procesos de identificación. De manera que las preguntas no irían referidas a los grupos de pertenencia, sino a la autoproducción de los mismos. Los individuos de acuerdo con las categorías dominantes usan estrategias de auto-identificación y de identificación de los otros en la medida que favorezcan su posición en las interacciones,

\footnotetext{
${ }^{5}$ A finales de la década de 1980 el tema de la ambivalencia (término acuñado por la psicología) empieza a atraer la atención de los sociólogos, acompañada por la decadencia de las grandes teorías que no reconocían la validez de principios contrarios (ciencia objetiva frente a ciencia reflexiva). Merton (1980) establece una serie de problemas sociológicos que la disciplina no consigue resolver y que sirven de motor, no sólo para acuñar el término, sino como desarrollo de la sociología contemporánea.
} 
con el fin de tener acceso a aquello que más valoren, sea de carácter económico, simbólico o emocional.

Así, la ambivalencia permite un margen de libertad que enfrenta al sujeto con su propia capacidad de elección. Del "¿Qué soy?" al "¿Qué quiero ser?", del "¿De dónde soy?" al "¿De dónde me siento?". Los alumnos viven esa experiencia, la de cuestionar o al menos preguntarse sobre el significado de un término como el de nacionalidad y producir formas de adhesión diferentes. El revuelo ocasionado ocupó gran parte de los debates posteriores dedicados a esta actividad y supuso una experiencia sociológica relevante y precursora de la ambigua relación que se mantendría a lo largo del curso escolar entre la interculturalidad, tal y como la trabajan la instituciones, y la realidad experimentada por los escolares. A pesar de esta experiencia, que traté de transmitir una y otra vez en una reunión con los profesores y miembros de la ONG para relativizar los resultados, éstos, a pesar de encontrar interesante el relato de lo sucedido, analizaron los resultados sociométricos finales sin más consideraciones, aplicando a los sujetos la nacionalidad jurídica correspondiente.

Al igual que sucede en estas escuelas con aulas multinacionales, Rafael Pulido retrata la dificultad de contabilizar y definir las culturas presentes en determinadas aulas donde la tutora tiene que responder a la pregunta que le formula un inspector educativo durante una visita al centro:

Entonces, ¿cuántas culturas distintas tienes en tu clase? ¿Qué debe hacer la maestra para responder apropiadamente? ¿Elegir una variable como principal marcador de diferencia entre culturas, por ejemplo, la nacionalidad, o, multiplicar las distintas categorías - nacionalidad, religión, lenguas maternas y características fenotípicas- y afirmar que en su clase hay representantes de doce de las cuatrocientas combinaciones culturales posibles?

Un problema similar se plantea con los resultados sociométricos. ¿Se debe tomar en consideración la nacionalidad objetiva (jurídica) de cada alumno, ignorando las consideraciones e identificaciones subjetivas, o superponer a la misma la nacionalidad subjetiva y/o atribuida por los propios sujetos?

El problema no reside tanto en la respuesta como en la pregunta, contabilizar y clasificar las culturas y las nacionalidades sin tener en consideración las identificaciones de los propios sujetos no recoge la complejidad de la realidad. Como apunta Besalù, la identidad personal tiene algunos elementos genéticos (sexo, algunos rasgos físicos) pero la mayoría de ellos son construidos y cambiantes a lo largo de nuestra existencia: "religión, nacionalidad, clase social, lengua, [...]. Somos el resultado de nuestras identificaciones: el ser social se construye en relación a los otros y a través de este juego de pertenencias" (Besalú 2002: 32).

¿Cómo implementar la interculturalidad cuando los sujetos escapan de las categorías necesarias para su desarrollo? Las categorías ejercen poder oscureciendo las diferencias y forzando lo múltiple en unidades manejables con sólidas separaciones, ya que sin ellas la acción se torna imposible (Jones 2009).

\section{CONCLUSIONES}

Hablar de interculturalidad en situaciones de inmigración se ha convertido en una forma de identificar la diversidad a través de su simplificación. Partir de las naciona- 
lidades de los estudiantes es reflejo de un intento de "ordenar" la diversidad y caracterizarla en bloques claramente diferenciados a partir de los cuales fomentar el reconocimiento e intercambio intercultural. El sociograma manifiesta cómo la vinculación de unos principios generales (la interculturalidad) con situaciones cotidianas y casos concretos hace emerger las contradicciones de un sistema de representación basado en la necesidad de nombrar y categorizar a los sujetos más allá de las identificaciones que operan en los mismos. El sociograma es, en efecto, una herramienta que produce categorías y sus relaciones, construyendo o conformando una determinada realidad social con base en las mismas. Necesita simplificar la realidad y agruparla en categorías y conceptos previamente definidos para poder operar sobre ella. Sin embargo, tal y como hemos podido comprobar, si el sociograma clasifica, el individuo se identifica.

La experiencia del sociograma ha permitido arrojar luz sobre los múltiples significados de la nacionalidad como concepto ambivalente en el que coexisten concepciones jurídicas, subjetivas y sociales. Ambivalencia que la ONG y los centros escolares no comparten, dando por hecho, uno, que la identificación de los sujetos pasa por la nacionalidad, y dos, que la nacionalidad es una realidad unívoca para todo el mundo. Estas asunciones son la fuente principal de conflicto que acompañan la interculturalidad como proyecto educativo tal y como es aplicado por las instituciones.

La interculturalidad se está construyendo mediante prácticas de intervención que toman la forma de actividades educativas y pedagógicas, transmitiendo determinadas categorías o representaciones de "lo deseable». En estas prácticas de intervención cotidiana y en la lógica de la que subyace a las mismas no hay verdaderos sujetos - contradictorios, internamente diversos, capaces de crítica, resistencia y lecturas alternativas a los discursos proyectados sobre ellos-, sino categorías sociales y posibles relaciones entre categorías. A través del sociograma, la ONG construye modelos de afiliación nacional o cultural "pasando por 'reales' cuando no son más que construcciones formales" (De Rudder 1997: 21). De esta forma, las categorías nacionales se imponen a los alumnos como dimensión en la percepción de sí mismos y de los demás. "Esta identificación por categorizaciones impone a los individuos la manera en la que deben contemplar sus propias vidas. Se les asigna un lugar, aunque no sea el que desean o reivindican" (Dubois 2003: 18-19). Los conflictos personales, problemáticas académicas y demás experiencias escolares terminan siendo mediatizados, interpretados y solucionados a partir de una interpretación nacional. Esta tendencia tiene el riesgo no ya de moldear las experiencias sociales, sino también de ocultar prácticas que pueden contradecirlas.

¿Qué ocurriría si en lugar de partir de las categorías nacionales establecidas por el sociograma partiéramos de las reacciones de los estudiantes ante las categorías propuestas y observásemos cómo los propios individuos se posicionan con respecto a las mismas y crean sus propios mundos de sentido? La experiencia del sociograma apunta la necesidad de explorar, en paralelo, más allá de la mirada del clasificador, las reacciones de los estudiantes y los vínculos reales que operan. Las resistencias con las que se ha encontrado este programa de intervención social muestran las paradojas de la interculturalidad como proyecto sociopolítico y apunta la necesidad de realizar un análisis crítico del proceso de categorización de los hijos de familias inmigrante que está teniendo lugar. 


\section{BIBLIOGRAFÍA CITADA}

Alegre Canosa, M. S. 2008. "Geografías adolescentes y contacto intercultural. Una visión desde el ámbito escolar". Papers 90: 33-98.

Allport, G. 1954. The Nature of Prejudice. Cambridge MA: Addison-Wesley.

Arnáiz, P. y De Haro, R. 2003. "Alumnos magrebíes en las aulas: analizar y comprender el presente para transformar y mejorar el mañana”. Educación, Desarrollo y Diversidad 6 (3): 63-82.

Aronson, E. y González, A. 1988. "Desegregation, jigsaw and the Mexican-American experience", En Katz, P. y Taylor, D. (eds.), Eliminating racism. Nueva York: Plenum.

Arruga, A. 1983. Introducción al test sociométrico. Barcelona: Herder.

Austin, J. L. 1962. How to do things with words. Oxford: Oxford University Press.

Ballesteros Velázquez, B. y Gil Jaurena, I. (coords.). 2012. Diversidad cultural y eficacia de la escuela. Un repertorio de buenas prácticas en centros de educación obligatoria. Madrid: Ministerio de Educación.

Banks, J.A. 2006. "Improving Race Relations in Schools: From Theory and Research to Practice". Journal of Social Issue 62 (3): 607-614.

Bauman, Z. 1990. Thinking Sociologically. Oxford: Blackwell.

Berry, J.W. 1997. "Immigration, acculturation, and adaptation". Applied Psychology 46 (1): 5-34.

Besalú, X. 2002. Diversidad cultural y educación. Madrid : Síntesis.

Bourdieu, P. y Wacquant, J. D. 1992. An invitation to reflexive sociology. Cambridge: Polity Press.

Cousin, O. 2002. "Les ambivalentes du travail. Les salariés peu qualifiés dan les centres d'appel". Sociologie du Travail 44: 499-520.

Criswell, J. H. 1939. "A sociometric structure of race cleavage in the classroom". Archives of Psychology 33 (235): 1-82.

De Federico de la Rúa, A. 2004. "Los espacios sociales de la transnacionalidad. Una tipología de la integración relacional de los migrantes". REDES-Revista hispana para el análisis de redes sociales 7 (4): 125-150.

De Rudder, V. 1997. "Quelques problèmes épistémologiques liés aux définitions des populations immigrantes et de leur descendance", en Aubert, F. et al. (eds.), Jeunes issus de l'immigration. París: L'Harmattan.

Del Olmo, M. 2010. Dilemas éticos en antropología. Las entretelas del trabajo de campo Etnográfico. Madrid: Trotta.

Díaz de Rada, A. 2013. "Etnografía de la escuela más allá de la etnografía y de la escuela: tensiones disciplinares y aplicabilidad de los saberes etnográficos". Educación y futuro:Revista de investigación aplicada y experiencias educativas 29: 13-39.

Dodge, K. A., Coie, J. D., Pettit, G. S., y Price, J. M. 1990. "Peer status and aggression in boys' groups: Developmental and contextual analyses". Child Development 61: 1289-1309.

Dubet, F. 2008. Faits d'école. París: Édition de l’École des Hautes Études en Sciences Sociales.

Dubois, V. 2003. La vie au guichet: relation administratie et traitrement de la misère. Paris: Económica.

Fernández Enguita, M.; Gaete, J. M. y Terrén, E. 2008. "¿Fronteras en las aulas? Contacto transcultural y endogamia en las interacciones del alumnado". Revista de Educación 345: 157-181.

Fernández Prados, J. S. y Soriano Ayala, E. 1997. "Realidad multicultural en las escuelas del poniente almeriense". Boletín del Instituto de Estudios Almerienses 7: 191-199.

Garay, A.; Íñiguez, L. y Martínez, L. M. 2003. "La perspectiva discursiva en psicología social”. Subjetividad y procesos cognitivos 7: 105-130.

García Castaño, F. J. y Carrasco, S. (eds.). 2008. Población inmigrante y escuela. Conocimientos y saberes de investigación. Textos reunidos en homenaje a Eduardo Terrén Lalana. Colección Estudios Creade, 5. Madrid: Ministerio de Educación.

García Castaño, F. J. y Olmos Alcaraz, A. (eds.). 2012. Segregación escolar y construcción de la diferencia en la escuela. Madrid: Trotta.

García Castaño, F. J. y Rubio Gómez, M. 2013. "Juntos pero no revueltos”: Procesos de concentración escolar del alumnado extranjero en determinados centros educativos". Revista de Dialectología y Tradiciones Populares LXVIII (1): 7-31. 
Gázquez Linares, J. J.; Pérez Fuentes, M. C.; Cangas Díaz, A. J. y Yuste Rossell, N. (coords.). 2007. Mejora de la convivencia y programas encaminados a la prevención e intervención del acoso escolar. Almería: Grupo Editorial Universitario.

Giménez, C. 2010. El interculturalismo: propuesta conceptual y aplicaciones prácticas. Bilbao: Observatorio Vasco de Inmigración.

Hewstone, M. y Brown, R. 1986. "Contact is not enough. An inter-group perspective on the "contact hypothesis", en Hewstone, M y Brown, R (eds.), Contact and Conflict in Intergroup Encounters. Oxford: Basil Blackwell.

Ibáñez, J. 1986. "Del pensamiento lineal al pensamiento complejo”. Alfoz 51: 13-18.

Jenkins, R. 2000. "Categorization: Identity, Social Process and Epistemology". Current Sociology 48 (3): $7-25$.

Jones, R. 2009. "Categories, borders and boundaries". Progress in Human Geography 33 (2): 174-189.

Kohli, M. 2000. "The battlegrounds of European Identity". European Societies 2 (2): 113-137.

Koster, M.; Nakken, H.; Pijl, S.J. y van Houten, E. 2009. "Being part of the peer group: A literature study focusing on the social dimension of inclusion in education". International Journal of Inclusive Education 13 (2): 117-140.

Lamo de Espinosa, E. 1989. Delitos sin víctima. Orden social y ambivalencia moral. Madrid: Alianza Universidad.

López Castro, G. 2000. "Richard y sus amigos. Sociometría de las relaciones en la escuela". Relaciones 21 (83): 121-138.

Martín, E. 2011. "The Influence of Diverse Interaction Contexts on Students' Sociometric Status". The Spanish Journal of Psychology 14 (1): 88-98.

Martínez Gutiérrez, P. 1999. "El sociograma como instrumento que desvela la complejidad”. Empiria, Revista de Metodología en Ciencias Sociales 2: 129-151.

Martínez Urralde, M. J. (ed.). 2010. “Y para muestra...políticas educativas de inmigración y modelos de escuela que practican la interculturalidad. Revista española de educación comparada 18: 371-373.

Merton, R. K. 1980. Ambivalencia sociológica y otros ensayos. Madrid: Espasa Calpe.

Moreno, J. L. 1954. Fundamentos de la Sociometría. Buenos Aires: Paidós.

Parsons, T. 1976. "La clase como sistema social: Alguna de sus funciones en la sociedad americana", en Gras, A (ed.). Textos fundamentales de la Sociología de la Educación. Madrid: Narcea.

Ponce Solé, J. 2007. Segregación escolar e inmigración contra los guetos escolares: Derecho y políticas públicas urbanas. Madrid: Centro de Estudios Políticos y Constitucionales.

Poveda, D.; Franzé, A.; Jociles, M. I.; Rivas, A.; Villaamil, F.; Peláez, C. y Sánchez, P. 2012. "La segregación étnica en la educación secundaria de la ciudad de Madrid: Un mapa y una lectura crítica", en García-Castaño, F. J. y Carrasco, S. (eds.), Población inmigrante y escuela: Conocimientos y saberes de investigación (CD-ROM). Madrid: IFIIE/CREADE.

Pulido, R. 2003. "Entonces, ¿̇cuántas culturas distintas tienes en tu clase?». Educación, desarrollo y diversidad 6 (3): 1-14.

Rovira Kaltwasser, C. 2012. "The ambivalence of populism: threat and corrective for democracy". Democratization 19 (2): 184-208.

Solbes, I. et al. 2011. "El contacto interétnico y su influencia sobre los prejuicios étnicos a lo largo de la niñez". Anales de Psicología 27 (3): 670.

Tedesco, J. C. 1995. El nuevo pacto educativo. Educación, competitividad y ciudadanía en la sociedad moderna. Madrid: Anaya.

Van Den Heuvel, H. y Meertens, R. W. 1989. "The culture assimilated, it is possible to improve interethnic relations by emphasizing ethnic differences?", en Van Oudenhoven, J. P y Wilemsen, T. M. (eds), Ethnic Minorities. Amsterdam: Swets y Zitlinger.

Fecha de recepción: 4 de octubre de 2012

Fecha de aceptación: 13 de marzo de 2014 